\title{
Video Denoising Using Fuzzy-connectedness Principles
}

\author{
Wing-kuen Ling and P. K. S. Tam \\ Department of Electronic and Information Engineering \\ The Hong Kong Polytechnic University \\ Hung Hom, Kowloon, Hong Kong \\ Hong Kong Special Administrative Region, China \\ Tel: (852) 2766-6238, Fax: (852) 2362-8439 \\ Email: bingo@encserver.eie.polyu.edu.hk, enptam@polyu.edu.hk
}

\begin{abstract}
Fuzzy-connectedness principles are effective for image segmentation. In this paper, such a principle is applied to video denoising. Assume a video signal suffers from both additive white Gaussian noise and impulsive noise. The corrupted signal is filtered by a fuzzy system, which fuzzily connects a Wiener filter and a median filter together. The simulation results show that the fuzzy-connectedness approach produces desirable outputs.
\end{abstract}

Index Terms - fuzzy-connectedness principles, image segmentation, video denoising, additive white Gaussian noise, impulsive noise, Wiener filter, median filter

\section{INTRODUCTION}

Noise is usually corrupted into a video signal during the transmission. Hence, video denoising plays an important role in research this decade.

An existing method for video denoising is subband denoising [1]. A video signal is required to break down into several subbands, and denoising techniques are applied on each subband. The computation complexity is too high for real-time video signal processing.

A hybrid Wiener-median filter is also proposed [2, 3]. However, a high percentage of the corrupted signal is fed forward to the output, and the result is undesirable.

Fuzzy approaches are suggested in $[4,5]$. But those methods employ a center average defuzzifier, which acts as a lowpass filter. The output is blurred and the image details are destroyed.

In this paper, a fuzzy-connectedness approach is proposed. Since a simple lowpass filter, such as a Wiener filter, is effective for additive white Gaussain noise (AWGN), while a nonlinear filter, such as median filter, is good for impulsive noise, both filters are employed in the fuzzy system. The problem is how to integrate these two filters. It is found that a fuzzy connection among these two filters gives a desirable result.

Fuzzy-connectedness principles are reviewed in Section II and similar principles for system connections are derived in Section III. Based on the derived principles, a video denoising algorithm which employs fuzzy-connectedness principles is proposed in Section IV. Simulation results are shown in Section V and a conclusion is given in Section VI.

\section{REVIEW ON FUZZY-CONNECTEDNESS PRINCIPLES}

Fuzzy-connectedness principles are widely applied to image segmentation [6,7]. Two pixels in an image, $a$ and $b$, are said to be fuzzily connected in a set $\mathbf{A}$ if there exists a fuzzy membership value $\mu_{A}(a, b) \in\left(\begin{array}{ll}0 & 1)\end{array}\right)$.

\section{FUZZY CONNECTION OF SYSTEMS}

For the fuzzy-connectedness principles applied to image segmentation, there is no real connection among the pixels. The fuzzy membership value is a measure of how two pixels belong to the same object. However, in the case of system connections, there is a real connection among the signals in a system and signal conflict may occur. In order to work on this problem, adders are connected to the input and output of each sub-system. The model is as below:

Assume there are $N$ sub-systems $\mathbf{T}_{\mathbf{i}}$, where $i=1,2, \ldots, N$, with the input and the output of $\mathbf{T}_{\mathbf{i}}$ being $x_{i}$ and $y_{i}$, respectively. Let the input and output of the sub-systems, including the adders, be $u_{i}$ and $z_{i}$, respectively, as shown in figure 1 . Define a set $\mathbf{A}$ which is the collection of the signals $x_{i}$ and $y_{i}$. That is, $\mathbf{A}=\left\{x_{1}, x_{2}, \ldots, x_{N}, y_{1}, y_{2}, \ldots, y_{N}\right\}$. Let $a, b \in \mathbf{A}$. The signal $a$ is said to be fuzzily connected to $b$ in $\mathbf{A}$ if there exists a fuzzy membership value $\mu_{A}(a, b) \in(01)$.

The fuzzy connection is equivalent to connecting a signal $a$ to an amplifier with the gain $\mu_{A}(a, b)$, and then connect the output of the amplifier to the adder which corresponds to the signal $b$ as shown in figure 2. In general, the fuzzy connection of systems can be realized as a neural network, with neurons $\mathbf{T}_{\mathbf{i}}$.

For a general case, $\mu_{A}(a, b)=\mu_{A}(b, a)$ is not necessarily true. But, $\mu_{A}(a, a)$ should be zero for all $a$ in A. Otherwise, a signal conflict may occur in the adders.

Since fuzzy connection includes all the connections among $x_{i}$ and $y_{i}$, the traditional series, parallel and feedback connections are particular cases of fuzzy connection. 


\section{A. Traditional series connection}

If $\mathbf{T}_{\mathbf{i}}$ is connected to $\mathbf{T}_{\mathbf{j}}$ in series, that is, $z_{j}=\mathbf{T}_{\mathbf{j}}\left(\mathbf{T}_{\mathbf{i}}\left(u_{i}\right)\right)$ and $u_{j}=0$, the corresponding fuzzy membership values are all zero except $\mu_{\boldsymbol{A}}\left(y_{i}, x_{j}\right)=1$. Similarly, if $\mathbf{T}_{\mathbf{j}}$ is connected to $\mathbf{T}_{\mathbf{i}}$ in series and $u_{i}=0$, the corresponding fuzzy membership values are all zero except $\mu_{A}\left(y_{j}, x_{i}\right)=1$.

\section{B. Traditional parallel connection}

If $\mathbf{T}_{\mathbf{i}}$ is connected to $\mathbf{T}_{\mathbf{j}}$ in parallel, that is, $z_{i}=\mathbf{T}_{\mathbf{i}}\left(u_{i}\right)$, $z_{j}=\mathbf{T}_{\mathbf{j}}\left(u_{i}\right)$ and $u_{j}=0$, the corresponding fuzzy membership values are all zero except $\mu_{\boldsymbol{A}}\left(x_{i}, x_{j}\right)=1$.

\section{Traditional feedback connection}

If $\mathbf{T}_{\mathbf{j}}$ is feedback connected to $\mathbf{T}_{\mathbf{i}}$, that is, $z_{i}=\mathbf{T}_{\mathbf{i}}\left(u_{i}+\mathbf{T}_{\mathbf{j}}\left(z_{i}\right)\right)$ and $u_{j}=0$, the corresponding fuzzy membership values are all zero except $\mu_{A}\left(y_{i}, y_{j}\right)=1$ and $\mu_{A}\left(x_{j}, x_{i}\right)=1$.

\section{VIDEO DENOISING USING FUZZY-CONNECTEDNESS PRINCIPLES}

In our denoising system, a Wiener filter and a median filter are employed. If $\mathbf{T}_{\mathbf{1}}$ is a Wiener filter and $\mathbf{T}_{\mathbf{2}}$ is a median filter, respectively, then $\mathbf{A}=\left\{x_{1}, y_{1}, x_{2}, y_{2}\right\}$. There are a total of four elements in set $\mathbf{A}$. Hence, we have 16 connections among those elements and each connection is associated with a fuzzy membership value as below:

\begin{tabular}{|c|c|c|c|c|}
\hline & $x_{1}$ & $x_{2}$ & $y_{1}$ & $y_{2}$ \\
\hline$x_{1}$ & $\mu_{A}\left(x_{1}, x_{1}\right)$ & $\mu_{A}\left(x_{1}, x_{2}\right)$ & $\mu_{A}\left(x_{1}, y_{1}\right)$ & $\mu_{A}\left(x_{1}, y_{2}\right)$ \\
\hline$x_{2}$ & $\mu_{A}\left(x_{2}, x_{1}\right)$ & $\mu_{A}\left(x_{2}, x_{2}\right)$ & $\mu_{A}\left(x_{2}, y_{1}\right)$ & $\mu_{A}\left(x_{2}, y_{2}\right)$ \\
\hline$y_{1}$ & $\mu_{A}\left(y_{1}, x_{1}\right)$ & $\mu_{A}\left(y_{1}, x_{2}\right)$ & $\mu_{A}\left(y_{1}, y_{1}\right)$ & $\mu_{A}\left(y_{1}, y_{2}\right)$ \\
\hline$y_{2}$ & $\mu_{A}\left(y_{2}, x_{1}\right)$ & $\mu_{A}\left(y_{2}, x_{2}\right)$ & $\mu_{A}\left(y_{2}, y_{1}\right)$ & $\mu_{A}\left(y_{2}, y_{2}\right)$ \\
\hline
\end{tabular}

Table 1. Fuzzy membership values of proposed system

The whole system is realized as a neural network as shown in figure 3 .

The problem becomes how to decide on those fuzzy membership values. We propose to use the first frame of a video sequence to train those 16 coefficients and use the trained coefficients to reduce the noise for the subsequent frames of the video sequence.

\section{SIMULATION RESULTS}

A set of 100 frames of size 288x360 8-bit gray video sequence "Claire" is corrupted by AWGN with zero mean variance $=100$ and impulsive noise with density 0.1 . The video sequence is filtered through the proposed fuzzy system with a $3 \times 3$ Wiener filter and a $3 \times 3$ median filter. The quality of each frame of a video sequence is measured by the peak-signal-to-noise ratio (PSNR) and defined as follows:

$$
P S N R=20 \cdot \log _{10} \frac{255}{\sqrt{\frac{\sum_{i=1}^{288} \sum_{j=1}^{360}(x(i, j)-\hat{x}(i, j))^{2}}{288 \cdot 360}}}
$$

Although the first frame is used for training, it can be seen in figure 4 that the PSNR of other frames is almost the same as that of the first frame. The quality of the video sequence is shown in figure 5 . We can conclude from the results that the fuzzy-connectedness approach gives the best quantitative and qualitative results among the existing methods.

\section{CONCLUDING REMARKS}

In this paper, a video denoising algorithm using a fuzzy-connectedness approach is proposed. Since Wiener filter is effective for AWGN and median filter is good for impulsive noise, a fuzzy connection between these two filters gives a desirable result.

If the noise power of AWGN and the impulsive noise is altered due to the change of the transmission channel, we only need to train the new set of fuzzy membership values based on the first frame of the video sequence. This guarantees a desirable result.

In the case of the change of the noise nature, for example, Gaussianly distributed noise is changed to uniformly distributed noise; we need to change to another type of filter which is effective for uniformly distributed noise. Finally, we re-train the fuzzy membership values to get a desirable result.

\section{ACKNOWLEDGEMENT}

The work described in this letter was substantially supported by a grant from the Hong Kong Polytechnic University with account number G-V968.

\section{REFERENCES}

[1] K. Anandakumar and Saleem A. Kassam, "Nonlinear Filtering Using Generalized Subband Decomposition," IEEE International Conference on Image Processing ICIP, vol. 1, pp. 382-385, 1995.

[2] Lazhar Khriji and Moncef Gabbouj, "Median-rational Hybrid Filters," IEEE International Conference on Image Processing ICIP, vol. 2, pp. 853-857, 1998.

[3] Soon Young Park and Yong Hoon Lee, "Double Smoothing of Images Using Median and Wiener Filters," IEEE Transactions on Acoustics, Speech, and Signal Processing, vol. 37, No. 6, pp. 943-946, June, 1989.

[4] Dr Sos Agaian and Amit Sheth, "Class of Non Linear Filters based on Fuzzy Membership Functions," Conference of the North American Fuzzy Information Processing Society - NAFIPS, pp. 170-177, 1998.

[5] H. K. Kwan and Y. Cai, "Median Filtering Using Fuzzy Concept," IEEE. Proceedings of the 36th Midwest Symposium on Circuits and Systems, pp. $824-827$, vol. 2, 1993.

[6] J. K. Udupa, L. Wei, S. Samarasekera, Y. Miki, M. A. van Buchem and R. I. Grossman, "Multiple Sclerosis Lesion Quantification Using Fuzzy-Connectedness Pronciples," IEEE Transactions on Medical Imaging, vol. 16, No. 5, pp. 598-609, October, 1997.

[7] Silvana G. Dellepiane, Franco Fontana and Gianni L. Vernazza, "Nonlinear Image Labeling for Multivalued Segmentation," IEEE Transactions on Image Processing, vol. 5, No. 3, pp. 429-446, March, 1996. 


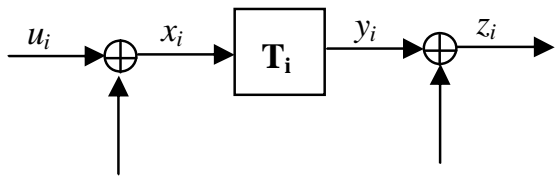

Fig. 1. Signals in a sub-system of a fuzzily connected system

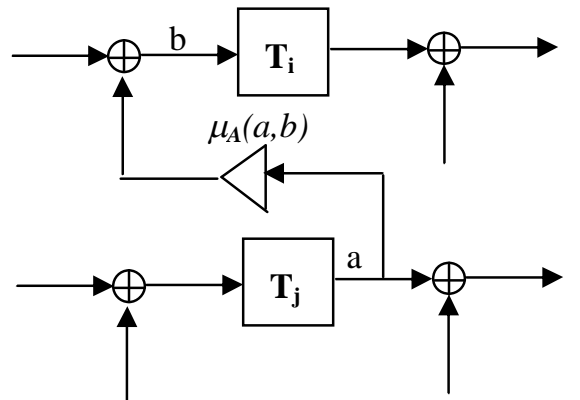

Fig. 2. Diagram demonstrates the physical connection of fuzzy membership value $\mu_{A}(a, b)$

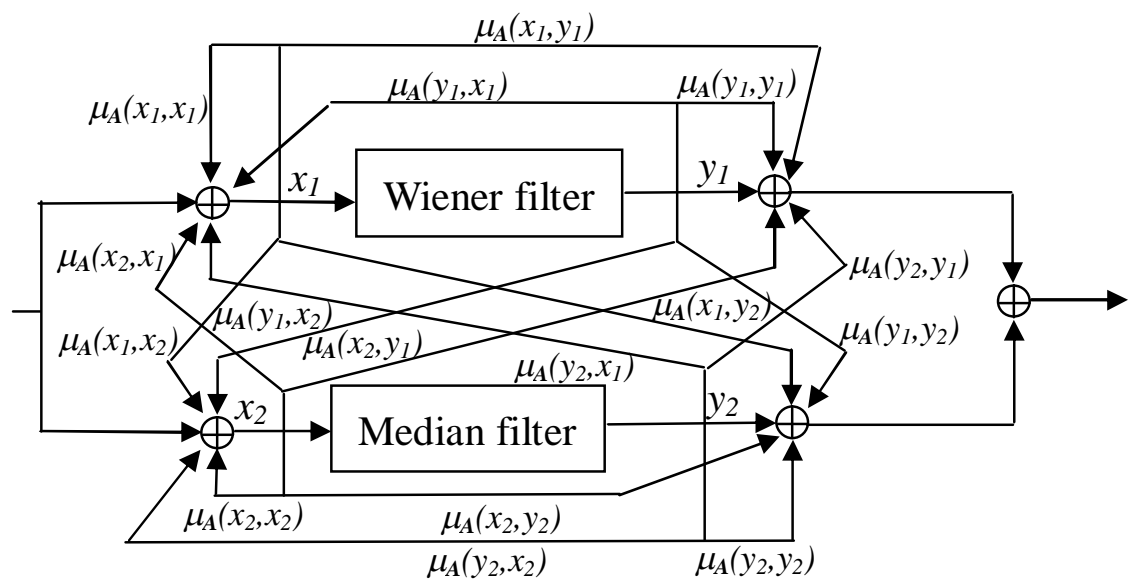

Fig. 3. Fuzzy-connectedness video denoising system

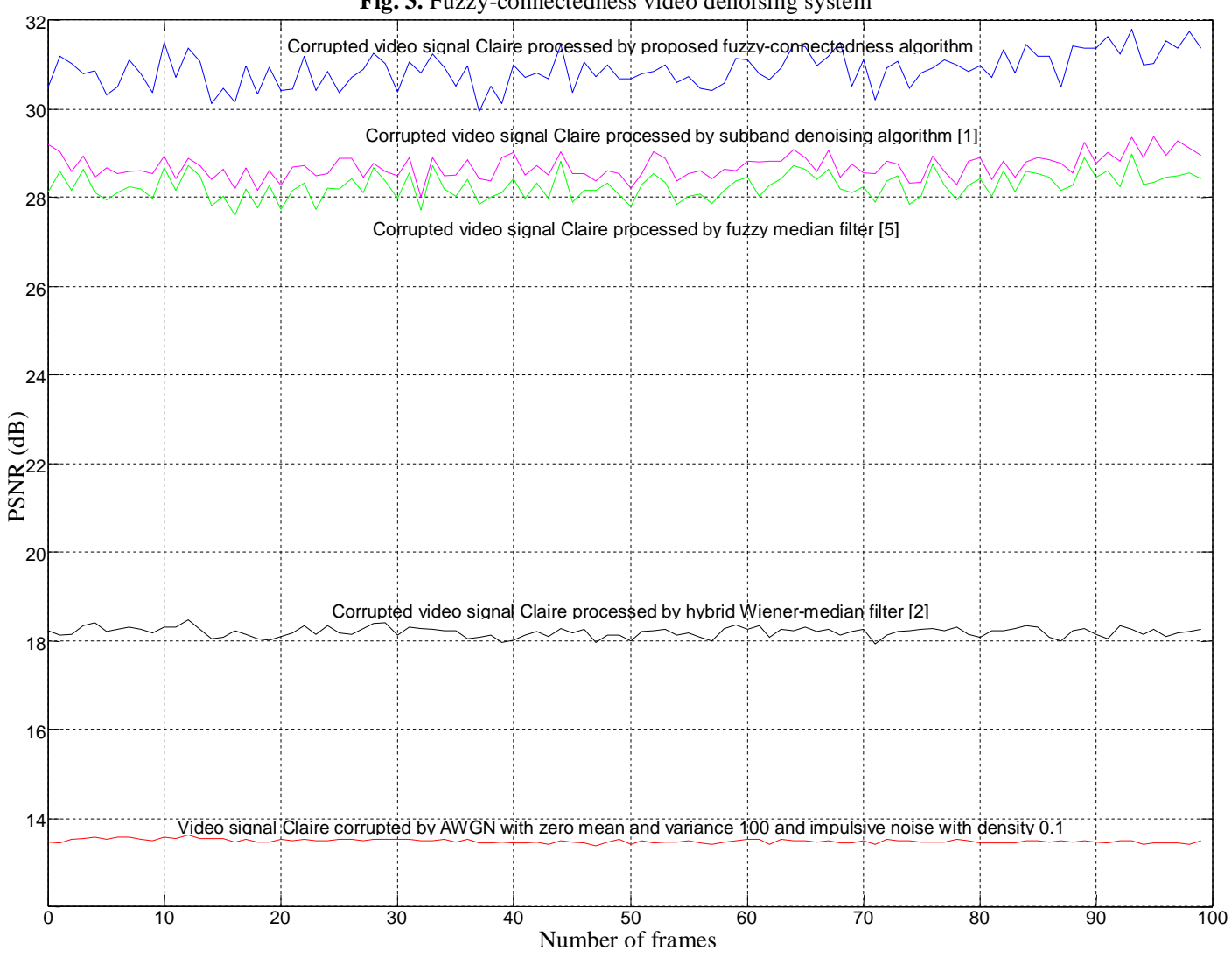

Fig. 4. Simulation results of corrupted video signal Claire processed by different algorithms 

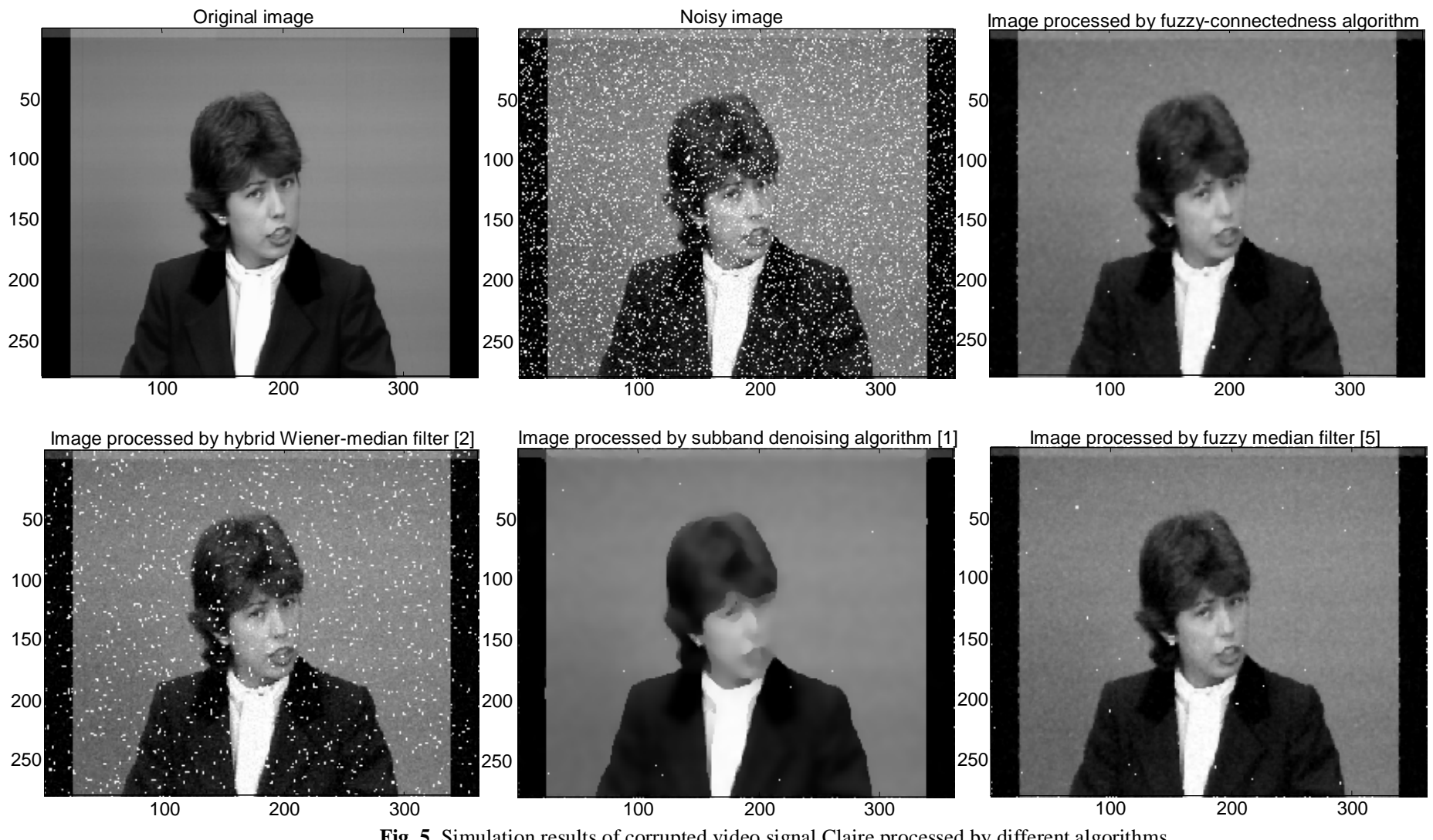

Fig. 5. Simulation results of corrupted video signal Claire processed by different algorithms 